\title{
May a correlation exist between age and biochemical parameters in Covid-19 patients?
}

\author{
Aslıhan Dilara Demir ${ }^{1}$, Zeynep Hulya Durmaz ${ }^{2}$ \\ ${ }^{1}$ Assistant Professor, Department of Internal Medicine. Amasya University Research Hospital, Amasya, Turkey, \\ ${ }^{2}$ Specialist, Department of Biochemistry, Kastamonu Research and Education Hospital, Kastamonu, Turkey
}

Background: Covid-19 infectious disease becomes a pandemic and later affects the entire world it has various effects in different age groups. Aims and Objective: In our study, we aimed to investigate the distribution of biochemical tests along with age, and the incidence in different age groups under the age of 65 and over the age of 65. Materials and Methods: We planned a retrospective study. Our patients consist of 2 groups. The first group consists of 54 patients aged 18 to 65 years, and the second group consists of 38 patients aged 65 years and older. Previously analyzed biochemical parameter values (such as lymphocyte, neutrophil, hemoglobin, platelet, CRP, LDH, AST, ALT, D-dimer, BUN, creatinine) of all patients followed up in our hospital were reached retrospectively through the hospital database. Results: There was a strong negative correlation between Alb and CRP, D-Dimer and albumin, neutrophil and albumin in patients under 65 years of age. A positive correlation was observed between other parameters. Negative moderate correlations were found between albumin and CRP, Potassium and age, WBC and albumin, hemoglobin and creatinine, hemoglobin and LDH, platelets and hemoglobin, lymphocytes and age, lymphocytes and prothrombin time, lymphocytes and D-Dimer, neutrophils and albumin, fibrinogen and creatine kinase, erythrocyte sedimentation rate and AST, erythrocyte sedimentation rate and ALT, erythrocyte sedimentation rate and hemoglobin in patients over 65 years of age. Positive correlations were detected between other parameters. Conclusion: ALT, creatinine, CRP, LDH, D-Dimer, WBC, neutrophil, erythrocyte sedimentation rate was significantly higher in patients over 65 years of age than in patients under 65 years of age. Platelet, lymphocytes were statistically significantly higher in patients under 65 than in patients over 65.

Access this article online

Website:

http://nepjol.info/index.php/AJMS

DOI: 10.3126/ajms.v12i7.37193

E-ISSN: 2091-0576

P-ISSN: 2467-9100

Copyright (c) 2021 Asian Journal of Medical Sciences

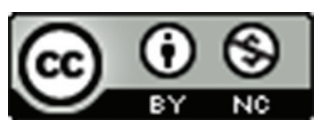

This work is licensed under a Creative Commons Attribution-NonCommercial 4.0 International License.

Keywords: Covid-19; Age; Lactate dehydrogenase (LDH); D-Dimer; Lymphocyte

\section{INTRODUCTION}

Coronaviruses are one of the major groups of viruses that affect the respiratory system. Our first encounter with coronaviruses as a pandemic was with SARS in 2002 and MERS in 2012.1,2 The covid-19 disease was identified in Wuhan in China in December 2019. It is an infectious disease that becomes a pandemic and later affects the entire world. ${ }^{3-5}$

Coronaviruses are RNA viruses divided into 4 groups: Alpha, beta, delta, and gamma (6). $2019 \mathrm{nCoV}$ is one of the beta viruses. ${ }^{4-7} \mathrm{~A}$ study conducted in China has shown that the disease is rare in children and that men get the disease more easily than women. They showed that CRP, D-Dimer levels increased, the number of leukocytes did not change, and the number of lymphocytes decreased. ${ }^{8}$

The disease can occur with fever, dyspnea (shortness of breath), dry cough, fatigue. It may occur in a form where the symptoms of respiratory infection are fainter and the symptom of diarrhea is at the forefront. ${ }^{9}$ Kai Lu et al., ${ }^{10}$ examined 18 elderly, 38 middle-aged, and young patients. They observed a higher mortality rate in the older group. As laboratory outcomes, they reported that the number of white blood cells and neutrophils was significantly higher in the elderly group. Again, they showed that the number of lymphocytes was lower in the elderly group. 
Just as the symptoms of the disease vary, it has different effects in different age groups. We aimed to examine, interpret and compare the laboratory outcomes of patients in these different age groups. It is hoped that the study will be beneficial to the better recognition and treatment of this new infectious disease.

\section{MATERIALS AND METHODS}

Our study is a retrospective study. The Ethics Committee of the Deaconship of the Faculty of Medicine of Tokat Gaziosmanpasa university approved the study with the registration number of 20-KAEK-217 dated 13.08.2020. Patients diagnosed with Covid-19 in our hospital were included in the study. Ninety-two patients over the age of 18 were included. These patients were retrospectively examined. Patients admitted with cough, fever, or upper respiratory tract complaints and followed up with Covid-19 diagnosis according to PCR and tomography results were included in the study. Examinations of patients were obtained through the hospital database. Previously analyzed biochemical parameter values (such as lymphocyte (LYM), neutrophil (NEUT), hemoglobin ( $\mathrm{Hb}$ ), platelet (PLT), C-Reactive protein (CRP), Lactate Dehydrogenase (LDH), Aspartate Aminotransferase (AST), Alanine Aminotransferase (ALT), D-Dimer, Bun, creatinine) of all patients followed up in our hospital were reached retrospectively through the hospital database. Patients were then divided into two groups. The first group consists of 54 patients between the ages of 18 and 65 . The second group consists of 38 patients aged 65 and over. Biochemical values of patients in these age groups were compared. Being under the age of 18 is the criteria for exclusion from the study.

In our hospital, $\mathrm{Hb}$, PLT, NEUT, and LYM values are analyzed with Sysmex XN 1000 hemogram device. The laser optical method is used. CRP is determined with Roche Cobas 8000 device by the turbidimetric method. The biochemical device is Roche Cobas 8000 .

GraphPad Prism version 8.00 was used for statistical analysis. (GraphPad Software, La Jolla California USA). After calculating quantitative data in Covid-19 positive patients over 65 years of age and under 65 years of age, the existence of the relationship between the variables was investigated by the Spearman correlation test. Correlation coefficient ( $\mathrm{r}$ ) was evaluated as weak from 0.00 to 0.24 , medium from 0.25 to 0.49 , strong from 0.50 to 0.74 , very strong from 0.75 to 1.00. Parameters measured in Covid-19 positive patients over 65 years of age and under 65 years of age were compared with a non-parametric test (MannWhitney test). Results were performed in the confidence interval of $95 \%$ with statistical significance $\mathrm{p}<0.05$.
Correlation coefficient $(\mathrm{r})$ was evaluated as weak from 0.00 to 0.24 , moderate from 0.25 to 0.49 , strong from 0.50 to 0.74 , very strong from 0.75 to 1.00 .

\section{RESULTS}

There was a very strong positive correlation between Neutrophil (NEUT) and WBC, ALT and AST, LYM and creatine kinase (CK), NEUT and CRP, fibrinogen (FGN) and CRP, erythrocyte sedimentation rate (ESR) and CRP, ferritin (FER) and lactate dehydrogenase (LDH), platelet (PLT) and WBC, FGN and WBC, FGN and NEUT, FGN and FER, ESR and FGN.

Also, moderate positive correlation was observed between CRP and age, CRP and AST, LDH and AST, LDH and creatinine, $\mathrm{K}$ and creatinine, $\mathrm{LDH}$ and $\mathrm{K}$, prothrombin time (PT) and $\mathrm{K}$, activate partial thromboplastin clotting time (APTT) and AST, APTT and CRP, D-dimer and CRP, WBC and CRP, WBC and LDH, WBC and $\mathrm{K}, \mathrm{WBC}$ and D-Dimer, Hemoglobin $(\mathrm{Hb})$ and ALT, PLT and Potassium (K), LYM and WBC, NEUT and LDH, NEUT and K, NEUT and D-Dimer, NEUT and PLT, NEUT and LYM, FER and age, FER and AST, FER and ALT, FER and creatinine, FER and CRP, FER and APTT, FER and D-Dimer, FER and WBC, FER and NEUT, FGN and age, FGN and AST, FGN and APT'T, FGN and D-Dimer, ESR and age, ESR and AST, ESR and LDH, ESR and NEUT, ESR and FER.

A strong negative correlation was found between Albumin (ALB) and CRP, D-Dimer and ALB, NEUT and ALB, also, a moderate negative correlation was observed between ALB and age, $\mathrm{Na}$ and age, $\mathrm{Na}$ and $\mathrm{AST}, \mathrm{Na}$ and $\mathrm{CRP}, \mathrm{Na}$ and LDH, APTT and ALB, APTT and Na, WBC and ALB, FER and ALB, FER and Na, FGN and ALB, FGN and $\mathrm{Na}, \mathrm{ESR}$ and ALB, ESR and $\mathrm{Na}, \mathrm{ESR}$ and $\mathrm{Hb}$.

ALT creatinine, CRP, LDH, D-Dimer, WBC, NEUT, ESR were statistically significantly higher in patients over 65 years of age than in patients under 65 years of age (Table 1).

$\mathrm{Pt}, \mathrm{LYM}$ were found to be significantly higher in patients under 65 years of age compared to patients over 65 years of age (Table 1).

In line with other studies conducted on this subject, lymphocyte levels in patients over the age of 65 were lower compared to young patients. ${ }^{8}$

Correlation coefficient $(\mathrm{r})$ was evaluated as weak from 0.00 to 0.24 , moderate from 0.25 to 0.49 , strong from 0.50 to 0.74 , very strong from 0.75 to 1.00 . 


\begin{tabular}{|c|c|c|c|}
\hline Parameters & Under $65(n=54)$ & Over $65(n=38)$ & $p$-value \\
\hline$\overline{\text { AST }}$ & $27,41 \pm 1,716$ & $41,84 \pm 9,88$ & 0,5442 \\
\hline ALT & $22,97 \pm 1,504$ & $23,53 \pm 4,957$ & $0,0074 *$ \\
\hline CREA & $0,855 \pm 0,03$ & $1,622 \pm 0,233$ & $0,0005^{*}$ \\
\hline CK & $106,5 \pm 13,58$ & $159,9 \pm 41,34$ & 0,5300 \\
\hline CRP & $37,70 \pm 5,782$ & $68,49 \pm 11,35$ & $0,0127 *$ \\
\hline ALB & $39,81 \pm 1,253$ & $38,25 \pm 4,579$ & $<0,0001$ \\
\hline LDH & $238,2 \pm 9,334$ & $310,4 \pm 41,53$ & $0,0270 *$ \\
\hline NA & $139,7 \pm 0,397$ & $139,6 \pm 0,99$ & 0,6176 \\
\hline K & $4,284 \pm 0,055$ & $4,194 \pm 0,102$ & 0,4005 \\
\hline PT & $17,33 \pm 2,68$ & $16,93 \pm 0,834$ & $0,0016 *$ \\
\hline APTT & $31,04 \pm 0,49$ & $33,24 \pm 1,37$ & 0,1070 \\
\hline D-Dimer & $0,917 \pm 0,183$ & $2,702 \pm 0,643$ & $<0,0001 *$ \\
\hline WBC & $6,910 \pm 0,441$ & $9,368 \pm 0,757$ & $0,0073 *$ \\
\hline $\mathrm{HB}$ & $13,50 \pm 0,253$ & $12,41 \pm 0,413$ & 0,0585 \\
\hline PLT & $216,1 \pm 11,93$ & $216,8 \pm 15,68$ & 0,9701 \\
\hline Lymphocyte & $5,969 \pm 3,71$ & $1,240 \pm 0,145$ & $0,0001 *$ \\
\hline Neutrophil & $4,298 \pm 0,385$ & $7,188 \pm 0,708$ & $0,0003 *$ \\
\hline Ferritin & $244,6 \pm 50,66$ & $270,2 \pm 47,14$ & 0,2806 \\
\hline Fibrinogen & $448,2 \pm 25,76$ & $483,3 \pm 30,49$ & 0,1806 \\
\hline $\begin{array}{l}\text { Erythrocyte } \\
\text { sedimentation rate }\end{array}$ & $35,18 \pm 3,57$ & $51,03 \pm 5,911$ & $0,0298 *$ \\
\hline
\end{tabular}

There were strong positive correlations between ALT and AST, LDH and AST, LDH and CK, NEUT and WBC, and strong positive correlation between CK and AST, LDH and ALT, PT and AST, PT and ALT, APTT and ALT, d-dimer and CRP, ESR and creatinine, FGN and CRP, FGN and d-dimer, ESR and FGN.

Also, there were moderate positive correlations between $\mathrm{CK}$ and ALT, CRP and age, $\mathrm{Na}$ and age, $\mathrm{K}$ and creatinine, PT, and CK, PT and CRP, PT and LDH, APTT and AST, APT'T and PT, D-Dimer and age, WBC and age, WBC and CRP, WBC and PT, PLT and WBC, LYM and PLT, NEUT and WBC, NEUT and CRP, NEUT and PT, FER and creatinine, FER and CRP, FER and K, FER and PT, FER and NEUT, FGN and creatinine, FGN and PT, FGN and WBC, FGN and NEUT, FGN and FER, ESR and CRP, ESR and D-Dimer, ESR and FER.

Negative moderate correlations were detected between $\mathrm{ALB}$ and $\mathrm{CRP}, \mathrm{K}$ and age, $\mathrm{WBC}$ and $\mathrm{ALB}, \mathrm{Hb}$ and creatinine, $\mathrm{Hb}$ and LDH, PLT and Hb, LYM and age, LYM and PT, LYM and D-Dimer, NEUT and ALB, FGN and CK, ESR and AST, ESR and ALT, ESR and Hb.

\section{DISCUSSION}

In our study, we aimed to investigate which biochemical tests such as white blood cells (WBC), NEUT, LYM, Hb, PLT, CRP, ESR, CK, AST, ALT, creatinine, D-Dimer, ALB, LDH, FER, FGN were higher or lower under the age of 65 and over the age of 65 . In this way, we aimed to draw attention to the biochemical parameters in the elderly group which is particularly affected by Covid-19 and has a poor prognosis. In the follow-up of at-risk individuals, we aimed to once again emphasize the importance of early and frequent blood checks in follow-up and treatment before the disease progresses.

In our study, ALT creatinine, CRP, LDH, D-Dimer, WBC, NEUT, and ESR were significantly higher in patients over 65 years of age than in patients under 65 years of age (Table 1).

PT, LYM were statistically significantly higher in patients under the age of 65 than in patients over 65 years of age (Table 1).

In patients under 65 years of age, strong negative correlations were observed between ALB and CRP, D-Dimer and ALB, NEUT and ALB, also, moderate negative correlations were observed between ALB and age, $\mathrm{Na}$ and age, $\mathrm{Na}$ and AST, $\mathrm{Na}$ and CRP, $\mathrm{Na}$ and $\mathrm{LDH}$, APTT and ALB, APTT and Na, WBC and ALB, FER and $A L B, F E R$ and $\mathrm{Na}$, FGN and ALB, FGN and $\mathrm{Na}, \mathrm{ESR}$ and ALB, ESR and Na, ESR and Hb. Between other parameters, positive correlations were observed (Figure 1).

Negative moderate correlations were found between Albumin and CRP, $\mathrm{K}$ and age, WBC and ALB, Hb and creatinine, $\mathrm{Hb}$ and LDH, PLT and Hb, LYM and age, LYM and PT, LYM and d-dimer, NEUT and ALB, FGN and $\mathrm{CK}, \mathrm{ESR}$ and AST, ESR and ALT, ESR and $\mathrm{Hb}$ in patients over 65 years of age. A positive correlation was observed between other parameters (Figure 2).

Xu X-W et al., ${ }^{11}$ conducted a study on patients who were followed in intensive care under the age of 60 and found leucopenia in $31 \%$ of patients and lymphopenia in $42 \%$, but the platelet count was normal and LDH was high in $27 \%$ of patients. AST, ALT, D-dimer, and $\mathrm{Hb}$ were within the normal limits.

Again, Huang $\mathrm{C}$ et al., ${ }^{9}$ conducted a study on patients with an average age of 49 and similarly found $63 \%$ lymphopenia, $25 \%$ leukopenia, and 5\% thrombocytopenia. LDH was high in $73 \%$ of patients. ALT, Hb, d-dimer were within normal limits.

In accordance with our study, Guan WJ et al., ${ }^{8}$ showed that lymphocyte levels over the age of 65 were lower compared to young patients.

Chan JF-W et al., ${ }^{12}$ found a low number of lymphocytes and platelets, while they showed an increase in CRP, LDH, PTT, and FGN. 


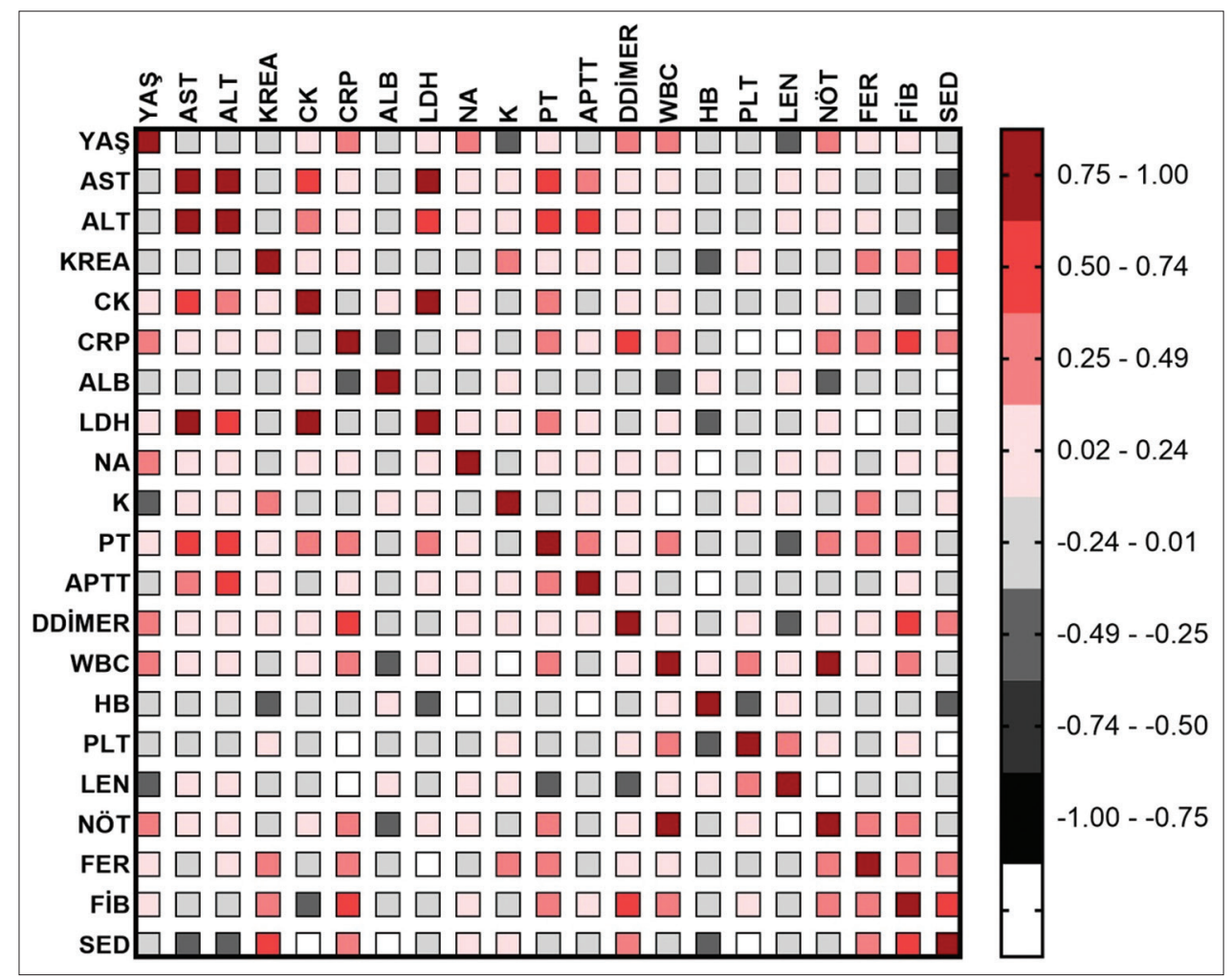

Figure 1: Correlation analysis of parameters of Covid-19-positive patients over 65 years of age

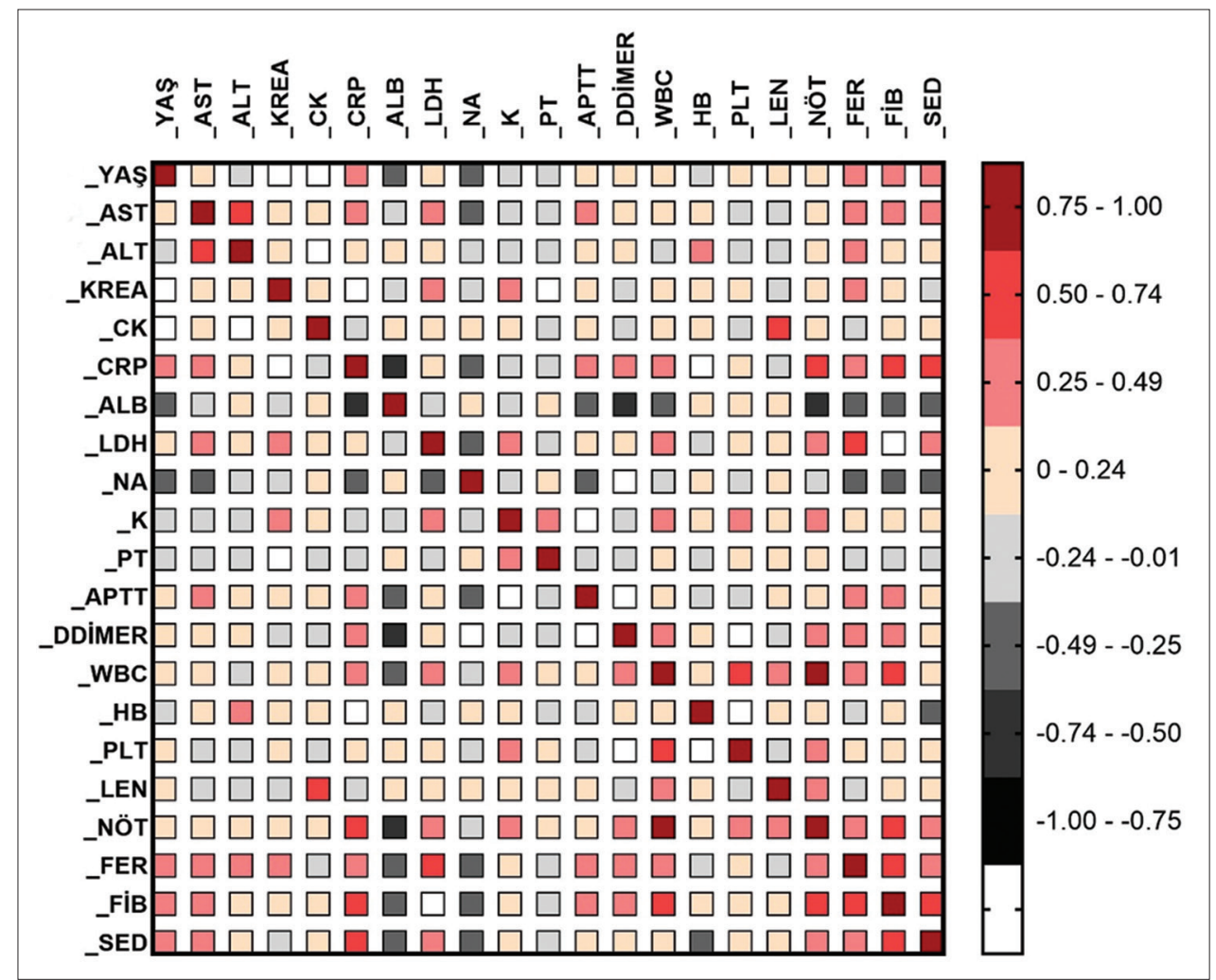

Figure 2: Correlation analysis of parameters of Covid-19-positive patients under 65 years of age

In the study of Liu Y et al., ${ }^{13} \mathrm{CRP}$ and LDH increased, also there was a decrease in LYM levels, and they did not detect changes in liver enzymes and platelets. In our study, we aimed to emphasize once again the importance of early 
and frequent blood checks in follow-up and treatment before the disease progresses, and the studies that will be done with more patients will shed light on this issue.

\section{ACKNOWLEDGMENTS}

First we would like to thank all the staff (doctors, nurses, laboratory staff) working in the management of this pandemic. We received no financial support. For this study, Tokat Gaziosmanpaşa University Research Hospital ethical board approval was taken.

\section{REFERENCES}

1. Rothan HA and Byrareddy SN. The epidemiology and pathogenesis of coronavirus disease (COVID-19) outbreak. J Autoimmun 2020;102433.

https://doi.org/10.1016/j.jaut.2020.102433

2. Desforges M, Le Coupanec A, Dubeau P Bourgouin A, Lajoie L, Dubé $\mathrm{M}$, et al. Human coronaviruses and other respiratory viruses: underestimated opportunistic pathogens of the central nervous system? Viruses. 2020; 12(1): 14.

https://doi.org/10.3390/v12010014

3. Zhou P, YangX-L, WangX-G, Hu B, Zhang L, Zhang W, et al. A pneumonia outbreak associated with a new coronavirus of probable bat origin. Nature. 2020;579(7798):270-273

https://doi.org/10.1038/s41586-020-2012-7

4. Wu F, Zhao S, Yu B, Chen Y-M, Wang W, Song Z-G, et al. A new coronavirus associated with human respiratory disease in China. Nature. 2020; 579:265-269.

https://doi.org/10.1038/s41586-020-2008-3

5. Lu R, Zhao X, Li J, Niu P, Yang B, Wu H, et al. Genomic characterization and epidemiology of 2019 novel coronavirus: implications for virus origins and receptor binding. Lancet. 2020; 395:565-574.
https://doi.org/10.1016/S0140-6736(20)30251-8

6. Fan Y, Zhao K, Shi ZL and Zhou P. Bat Coronaviruses in China. Viruses. 2019;11(3):210.

https://doi.org/10.3390/v11030210

7. Zhou P, Yang XL, Wang XG, Hu B, Zhang L, Zhang W, et al. Discovery of a novel coronavirus associated with the recent pneumonia outbreak in humans and its potential bat origin. bioRxiv preprint

https://doi.org/10.1101/2020.01.22.914952

8. Guan W, Ni Z, Hu Y, Liang W-H, Ou C-G, He J-X, et al. Clinical characteristics of 2019 novel coronavirus infection in China. medRxiv. 2020 Now published in New England Journal of Medicine.

https://doi.org/10.1056/NEJMoa2002032

9. Huang C, Wang Y, Li X, Ren L, Zhao J, Hu Y, et al. Clinical features of patients infected with 2019 novel coronavirus in Wuhan, China. Lancet. 2020; 395:497-506. https://doi.org/10.1016/S0140-6736(20)30183-5

10. Liua K, Chenb Y, Linc R and Han K. Clinical features of COVID-19 in elderly patients: A comparison with young and middle-aged patients. J Infect. 2020;80(6):e14-e18. https://doi.org/10.1016/j.jinf.2020.03.005

11. Xu X-W, Wu X-X, Jiang X-G, Xu K-J, Ying L-J and Chun- Ma $C-L$. Clinical findings in a group of patients infected with the 2019 novel coronavirus (Sars-Cov-2) outside of Wuhan, China: retrospective case series BMJ. 2020; 368. https://doi.org/10.1136/bmj.m606

12. Chan JFW, Yuan S, Kok KH, Wang To KK, Chu H, Yang J, et al. A familial cluster of pneumonia associated with the 2019 novel coronavirus indicating person-to-person transmission: a study of a family cluster. Lancet. 2020; 395:514-523. https://doi.org/10.1016/S0140-6736(20)30154-9

13. Liu $Y$, Yang $Y$, Zhang $C$, Huang $F$, Wang $F$ and Yuan J. Clinical and biochemical indexes from 2019-Ncov infected patients linked to viral loads and lung injury. Sci China Life Sci.2020;63(3):364-374. https://doi.org/10.1007/s11427-020-1643-8

Author's Contribution:

ADD-Concept and design of the study; prepared first draft of manuscript, coordination, review of literature and manuscript preparation; ZHD- Interpreted the results; reviewed the literature and manuscript preparation, Statistical analysis, interpretation and revision of the manuscript.

Work Attributed to:

Amasya University Research Hospital, Amasya, Turkey.

Orcid ID:

Dr.Aslıhan Dilara Demir- (10 https://orcid.org/0000-0002-3395-7533

Dr.Zeynep Hulya Durmaz- (1) https://orcid.org/0000-0002-3260-0030

Source of Funding: None, Conflict of Interest: None. 